\title{
Evaluation de l'activité du cadmium, en présence du zinc, sur les structures des tissus régulateurs du métabolisme chez le rat Wistar
}

\author{
Abdelkrim BERROUKCHE ${ }^{1, *}$, Miloud SLIMANI ${ }^{1}$, Khaled KAHLOULA ${ }^{1}$, Hafsa KAFI ${ }^{2}$ \\ et Amina CHEIKH ${ }^{2}$ \\ ${ }^{1}$ Laboratoire de Biochimie, Département de Biologie, Faculté des Sciences, \\ Université Tahar Moulay, Saida, Algérie. \\ ${ }^{2}$ Département de Biologie, Faculté des Sciences, Université Moulay Tahar, Saida, Algérie. \\ *Auteur correspondant ; E-mail: kerroum1967@yahoo.fr; berrouabd@gmail.com; (Adresse : 17 cité des \\ frères Moulay, Saida 20000, Algérie / Tél : 048518557 ; Tél : 0798520868).
}

\section{RESUME}

Le sulfate de Cadmium $\left(\mathrm{CdSO}_{4}\right)$ et le chlorure de $\mathrm{Zinc}\left(\mathrm{ZnCl}_{2}\right)$ ont des effets antagonistes. Le Cd est connu pour son effet nécrotique et le $\mathrm{Zn}$ pour son rôle protecteur. L'objectif de cette étude est d'évaluer les effets de l'exposition au Cd sur l'histologie du foie et des reins chez des rats Wistar en présence du $\mathrm{Zn}$. L'étude a porté sur 16 rats, âgés de 4 mois, qui ont été subdivisés en 4 groupes de 4 rats, le $1^{\text {er }}$ groupe recevait par voie orale du $\mathrm{Cd}(0,15 \mathrm{mg} / \mathrm{kg}$ de poids corporel) pendant un mois, le second groupe a été exposé au $\mathrm{Zn}$ à la même dose, un $3^{\mathrm{e}}$ groupe recevait un mélange $(\mathrm{Cd}-\mathrm{Zn})$ et le $4^{\mathrm{e}}$ groupe était le témoin recevant de l'eau de robinet dans les même conditions. Les résultats ont montré que l'exposition des rats au $\mathrm{Cd}$ a induit une diminution de leur poids corporel. L'examen histologique, chez les rats exposés au $\mathrm{Cd}$, a révélé des désorganisations cellulaires, des atypies cyto-nucléaires et des nécroses au niveau des tissus hépatiques et rénaux. Par ailleurs, les animaux ayant reçu du $\mathrm{Zn}$ ou un mélange $\mathrm{Cd}-\mathrm{Zn}$ ont préservé l'intégrité de l'organisation cellulaire et nucléaire de leur tissu. L'exposition au $\mathrm{Cd}$ était responsable de l'apparition d'importants changements morphologique et histologique chez le rat mais la présence du $\mathrm{Zn}$ a atténué partiellement les effets toxiques induits par le Cd.

(C) 2014 International Formulae Group. All rights reserved.

Mots clés : Cadmium, zinc, antagonistes, foie, reins, histologie.

\section{INTRODUCTION}

Le cadmium (Cd) est un métal lourd toxique et un polluant environnemental important présent dans le sol, l'eau, l'air, les aliments et la fumée de cigarette (WHO, 1992). L'intoxication par le Cd endommage différents tissus humains et animaux. Ce métal toxique est aussi cancérogène (Fernandez et al., 2003) et néfaste pour la reproduction en provoquant un retard de croissance et la stérilité (Fahmy and Aly, 2000). Au niveau du sang et des tissus, le $\mathrm{Cd}$ déclenche la formation d'entités d'oxygène réactif qui sont les radicaux libres de l'oxygène (ROOR ou peroxydes). Ces entités entraînent des dommages oxydatifs et la perte de fonctions physiologiques des membranes des érythrocytes et d'autres cellules (Liu et al., 
2009). Il est admis que la plupart des effets toxiques du $\mathrm{Cd}$ sont le résultat d'un déficit de traces d'éléments essentiels comme le zinc (Zn), qui est considéré comme un antioxydant (Lansdown et al., 2000). Les interactions du $\mathrm{Cd}$ avec le $\mathrm{Zn}$ peuvent avoir lieu à différentes étapes de l'utilisation de cet oligoélément par notre organisme (l'absorption, la distribution et l'excrétion) et cela peut ainsi affecter les fonctions biologiques du $\mathrm{Zn}$ (Lansdown et al., 2000). Le Zn est un excellent antioxydant qui empêche la synthèse des radicaux libres d'oxygène qui sont responsables du stress oxydative (Fernandez et al., 2003). Certaines études ont rapporté la capacité du $\mathrm{Zn}$ d'interagir avec les métaux tels que le cuivre et le fer et qui diminuent leurs concentrations dans différents tissus et inhibent les processus du stress oxydatif (Santon et al., 2003). Le Zn est impliqué dans la stabilisation de la membrane cellulaire, la synthèse de la métallo-thionéine (MT) et la structure de la super oxyde dismutase (SOD $\mathrm{Cu} / \mathrm{Zn}$ ) (Tandon et al., 2001). De nombreuses études ont montré que la supplémentation de l'alimentation en $\mathrm{Zn}$ peut réduire l'absorption et l'accumulation de $\mathrm{Cd}$ au niveau des tissus, et aussi, prévenir les actions néfastes du $\mathrm{Cd}$ (Brzoska et Moniuszko-Jakoniuk, 2000), alors qu'une carence en $\mathrm{Zn}$ peut favoriser l'accumulation du $\mathrm{Cd}$ et ainsi d'exercer sa toxicité (Liu et al., 2009). L'objectif de la présente étude est d'évaluer les effets toxiques $\mathrm{du} \mathrm{Cd}$ et ainsi que l'éventuel rôle protecteur $\mathrm{du} \mathrm{Zn}$ au niveau des tissus hépatiques et rénaux chez des rats Wistar.

\section{MATERIEL ET METHODES \\ Expérimentation animale \\ Traitements des animaux}

L'étude a été réalisée sur des rats adultes males, âgés de quatre mois, de souche «Wistar» élevés à l'animalerie du Département de Biologie, Faculté des
Sciences, Université de Saida. Leur poids corporel variait entre 83-242 g. L'élevage était effectué dans une pièce éclairée 12 heures par jour, il s'agissait d'une photopériode de $12 \mathrm{~h} / 24 \mathrm{~h}$, et dont la température était maintenue constante $\left(22\right.$ à $\left.25^{\circ} \mathrm{C}\right)$. Les 16 rats, utilisés dans cette étude, étaient logés dans des cages métalliques où chaque cage regroupait quatre rats. Ils avaient libre accès à l'eau et à la nourriture. Les animaux ont été traités conformément au principe et directive énoncés dans le manuel sur le soin et l'utilisation des animaux d'expérimentation (UPHS, 1985).

\section{Groupes d'animaux}

Les animaux ont été séparés en 4 groupes (Lot A, B, C et D) de 4 rats chacun :

- Lot A: Les rats recevaient quotidiennement par voie orale un volume de $1 \mathrm{ml}$ d'une solution de sulfate de cadmium $\left(\mathrm{CdSO}_{4}\right)$ à la même dose $(0,15 \mathrm{mg} / \mathrm{kg}$ poids corporel) pendant 30 jours.

- Lot B: Les rats recevaient chaque jour par gavage gastrique un volume de $1 \mathrm{ml}$ d'une solution de chlorure de zinc $\left(\mathrm{ZnCl}_{2}\right)$ à la même dose pendant 30 jours.

- Lot C: Les rats recevaient chaque jour par gavage gastrique un volume de $1 \mathrm{ml}$ constitué d'un mélange de $0,5 \mathrm{ml}$ de $\mathrm{ZnCl}_{2}$ et $0,5 \mathrm{ml}$ de $\mathrm{CdSO}_{4}$ à la même dose pendant 30 jours.

- Lot D: Les rats constituaient le groupe des témoins qui recevaient quotidiennement par gavage gastrique de l'eau de robinet pendant 30 jours.

L'ensemble des rats a fait l'objet de pesées hebdomadaires. Le gain ou la perte de poids des rats, soumis à l'étude, est la différence entre leur poids final et initial.

\section{Choix de la dose toxicologique}

La dose des sels de $\mathrm{Zn}$ et de $\mathrm{Cd}$, utilisée dans la présente étude, était de 0,15 $\mathrm{mg} / \mathrm{kg}$ poids corporel. Cette concentration a été utilisée comme dose pathologique ou 
toxicologique en référence à la dose modèle élaborée par l'équipe de Silvina et al. (2004), au cours de leur expérience sur les modifications morphologiques et le stress oxydatif opérés au niveau des différents tissus (foie, poumons et reins) exposés à une dose non carcinogène du $\mathrm{Cd}$.

Préparation des solutions des sels de zinc et de cadmium

Des quantités de $15 \mathrm{mg}$ de chacun des sels; $\mathrm{ZnCl}_{2}$ et $\mathrm{CdSO}_{4}$, ont été dissoutes séparément dans un même volume de $1000 \mathrm{ml}$ d'eau distillée pour préparer ces concentrations.

\section{Gavage}

A chaque rat a été administré, par voie orale, $1 \mathrm{ml}$ de solution pour $100 \mathrm{~g}$ de son poids corporel. La solution était renouvelée deux fois par semaine durant toute la période du traitement. Le gavage, réalisé par une sonde en acier inoxydable, était quotidien et avait lieu à la même heure pendant 30 jours. Ces animaux ont fait l'objet d'une surveillance pendant toute la période d'étude.

\section{Etudes histologiques}

A la fin de la période d'expérimentation, les rats témoins et expérimentés étaient âgés de plus de 4 mois. Ces animaux ont été sacrifiés par une technique exsangue en coupant l'artère brachiale après avoir été anesthésiés à l'éther. Différents organes à savoir; le foie et les reins ont été rapidement prélevés et conservés dans une solution de formol à $10 \%$. Ces tissus étaient destinés à des études macroscopique et microscopique. L'examen histologique a été effectué au niveau du Laboratoire de Pathologie du CHU de Sidi-Bel-Abbès. L'examen macroscopique des prélèvements fournit plusieurs indications à savoir ; la taille et la localisation de la partie lésée au niveau du tissu prélevé qui sera sélectionnée pour la prochaine étude. L'examen microscopique fait appel à une technique de base comportant différentes étapes à savoir ; Fixation, déshydratation, inclusion en paraffine, réalisation des coupes et colorations à l'Hématoxyline-Eosine (HE) suivie de l'observation au microscope optique à l'objectif 40.

\section{Analyses statistiques}

L'analyse statistique des données a été réalisée à l'aide du logiciel SIGMAPLOT version 3.1. Les résultats étaient exprimés par leurs moyennes affectées de leur déviation standard ( \pm DS). La comparaison des poids corporels moyens des animaux a été effectuée par le test $\mathrm{t}$ de Student et par la méthode d'analyse des variances ANOVA à une différence de significativité des résultats $\alpha \leq$ 0,05 .

\section{RESULTATS}

\section{Variation du poids corporel}

Le poids corporel des rats a été mesuré tous les 5 jours. Les mesures de poids ont montré des variations plus ou moins significatives $(p<0,05)$. Le poids corporel moyen, dans le groupe de rats exposés au $\mathrm{Cd}$, a été de $113 \pm 8,14 \mathrm{~g}$ alors que dans les autres groupes de rats, on a noté des valeurs différentes qui étaient de 125,42 $\pm 26,32 \mathrm{~g}$ pour le groupe de rats exposés au $\mathrm{Zn}, 172,28$ $\pm 15,33 \mathrm{~g}$ pour le groupe de rats exposés au mélange $\mathrm{Cd}-\mathrm{Zn}$ et $203,14 \pm 30.11 \mathrm{~g}$ pour le groupe de rats témoins (Figure 1).

\section{Etudes histologiques}

\section{Poids et dimensions des tissus prélevés}

Après la pesée des organes prélevés (foie et reins), une diminution du poids des organes et celle de leurs poids relatifs dans le lot A ont été constatées (Tableau 1). 


\section{Aspects et architecture des tissus prélevés}

\section{Tissu hépatique}

Une forte accumulation du $\mathrm{Zn}$ est suspecte au niveau du tissu hépatique à cause de la présence de la coloration grisâtre du parenchyme hépatique, expliquant ainsi l'augmentation du poids de l'organe. Le tissu hépatique présentait des kystes remplis de liquide séreux (Tableau 2). Aucune lésion ou nécrose apparente n'a été observée. Par contre, le tissu hépatique présentait des zones blanchâtres chez les rats du lot A (exposés au $\mathrm{Cd})$ qui seraient dues à la forte dose du $\mathrm{Cd}$ ou à une inflammation subaigüe ou lymphogranulomateuse.

Tissu rénal

Une faible diminution du poids du tissu rénal a été enregistrée chez les rats exposés au $\mathrm{Cd}$ et $\mathrm{Zn}$ par rapport à celui des reins chez les rats témoins, reflétant ainsi une faible accumulation du $\mathrm{Cd}$ et $\mathrm{Zn}$ dans le tissu rénal et un aspect normal de ce dernier (Tableau 2). La morphologie du tissu rénal n'a pas été affectée dans sa totalité et aucune lésion n'était apparente chez les rats du lot A (exposés au Cd) et ainsi que pour les autres lots de rats et cela pourrait être dû à la dose $(0,15 \mathrm{mg} / \mathrm{kg}$ poids corporel) choisie dans cette étude. Aucune zone œdémateuse ou une dilatation des tubules du tissu rénal n'a été observée. De même, il était noté une absence d'inflammation au niveau des capillaires.

\section{Examen microscopique}

Tissu hépatique

Les coupes de tissus hépatiques, prélevés chez les rats témoins, ont montré des plages d'hépatocytes normales (Figure 2 A). D'une autre part, il a été observé chez les rats exposés au $\mathrm{Cd}$ des hépatocytes ayant un cytoplasme avec une dégénérescence granulaire et vacuolaire et un noyau volumineux avec une hétéro chromatine claire et proéminente. Les cellules hépatiques sont élargies avec une forme sinusoïdale (Figure 2 B). Des lésions inflammatoires au niveau du tissu hépatique étaient fréquentes en cas d'exposition des rats au Cd (Figure 2 B). Ce même type de lésions renfermait des inflammations exsudatives et lymphogranulomateuses témoignant de la présence de kystes au niveau du tissu hépatique des rats du lot B, exposés au Zn (Figure 2 C). Cependant, les rats du lot $\mathrm{C}$ (recevant un mélange $\mathrm{Cd}-\mathrm{Zn}$ ) présentaient un tissu hépatique avec une organisation cellulaire semblable à celle du tissu hépatique des rats témoins du lot $\mathrm{D}$ (Figure 2 D).

Tissu rénal

Les rats témoins présentaient un tissu rénal avec des zones corticale et medullaire normales (Figure $3 \mathrm{~A}$ ). Le tissu rénal des rats, exposés au $\mathrm{Cd}$, montrait une dégénérescence tubulaire considérable avec une atteinte glomérulaire (Figure 3 B). L'organisation tissulaire présentait un épithélium tubulaire ayant une structure floue et une atrophie de la bordure en brosse. L'accumulation du Cd dans les cellules épithéliales entraîne leur fragmentation et la libération de noyaux dans la lumière tubulaire. Le cytoplasme des cellules épithéliales est plus ou moins granulaire. Les tubules cortico-medullaire sont endommagés. Le tissu rénal est alors détruit et remplacé par du tissu fibreux blanc. Par ailleurs, le tissu rénal des rats témoins ou des autres lots de rats (recevant du $\mathrm{Zn}$ ou du mélange $\mathrm{Cd}-\mathrm{Zn}$ ) ne présentait aucune désorganisation tissulaire (Figures $3 \mathrm{C}$ et $\mathrm{D}$ ). 
A. BERROUKCHE et al. / Int. J. Biol. Chem. Sci. 8(4): 1796-1807, 2014

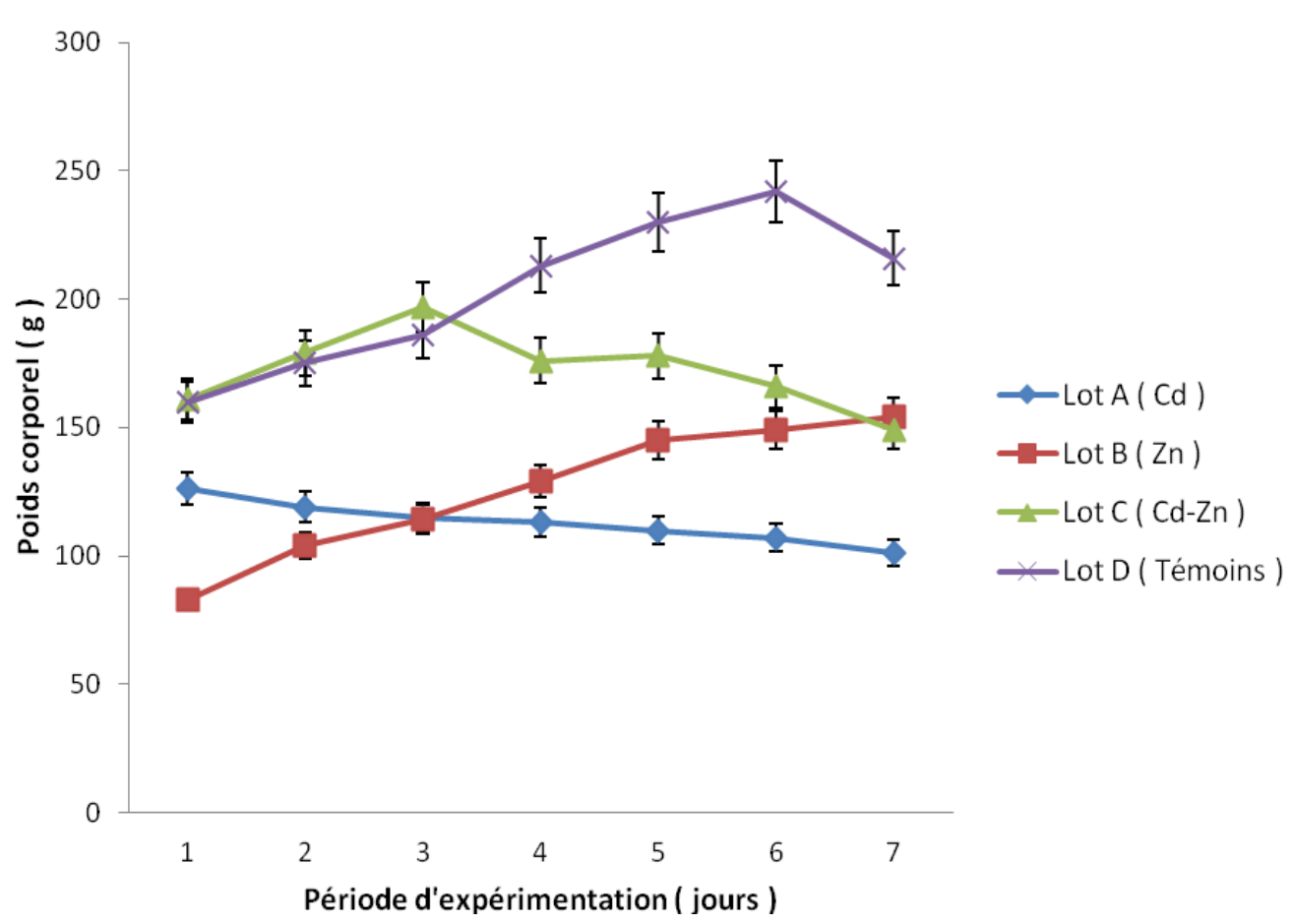

Figure 1 : Variation du poids corporel chez les rats exposés au Cd et Zn.

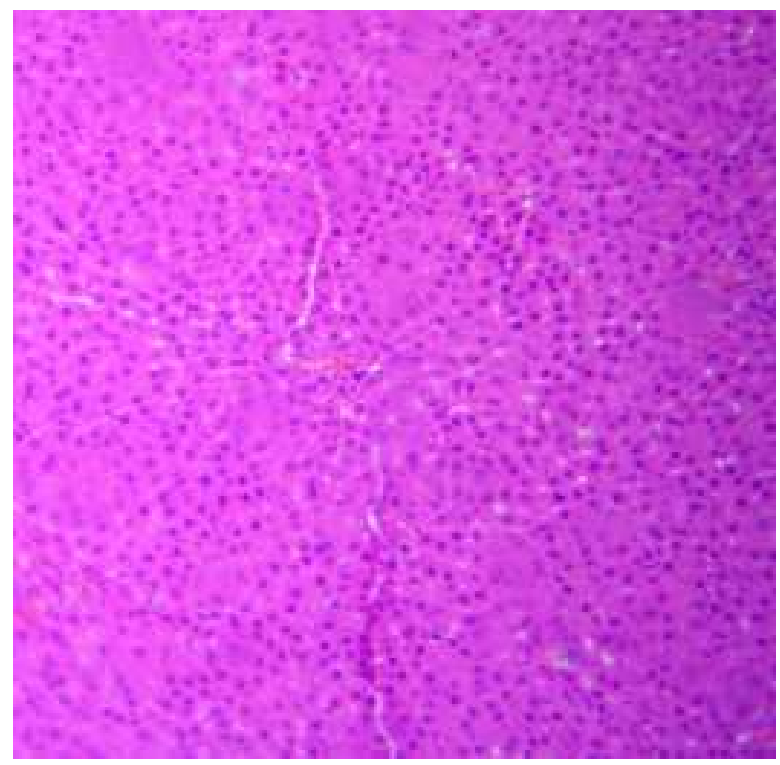

(A) : Lot A ; Tissu hépatique normal (Hematoxyline-Eosine $\times 40$ ). 


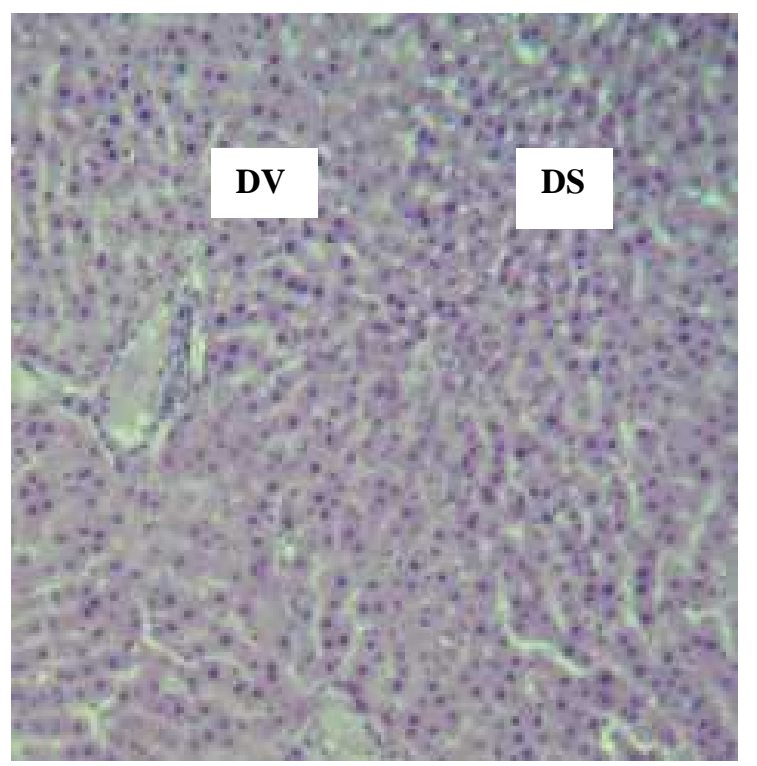

(B) : Lot B $\left(\mathrm{CdSO}_{4}\right)$; Dégénérescence vacuolaire et granulaire du cytoplasme [DV]-Dilatation sinusoïdale des cellules [DS] $(\mathrm{H}-\mathrm{E} \times 40)$.

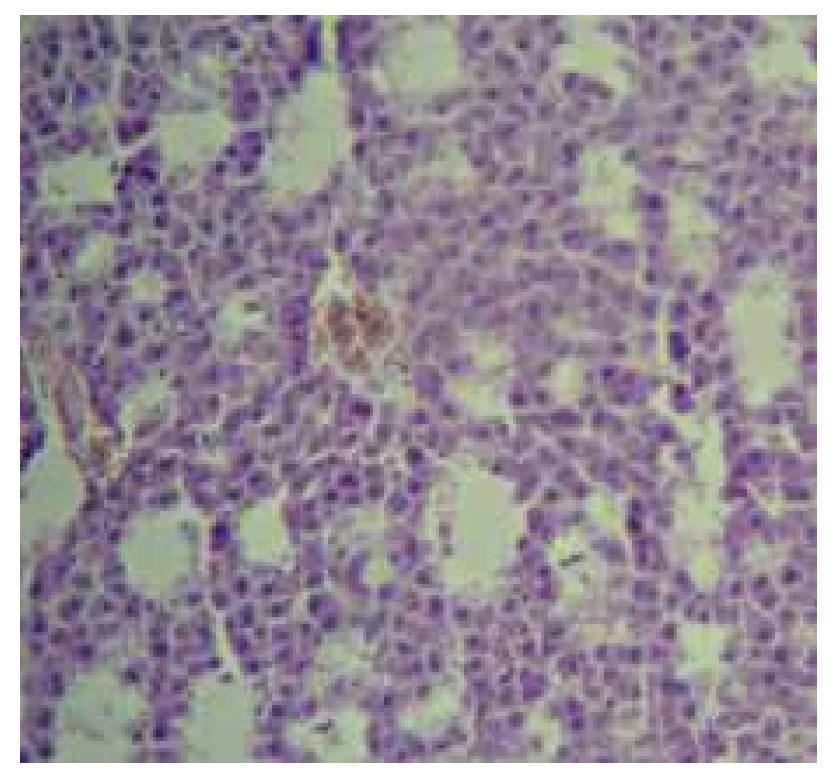

(C) : Lot $\mathrm{C}\left(\mathrm{ZnCl}_{2}\right)$; Lésions inflammatoires exsudatives [LIE] et lympho-granulomateuses [LLG] (H-Ex 40). 
A. BERROUKCHE et al. / Int. J. Biol. Chem. Sci. 8(4): 1796-1807, 2014

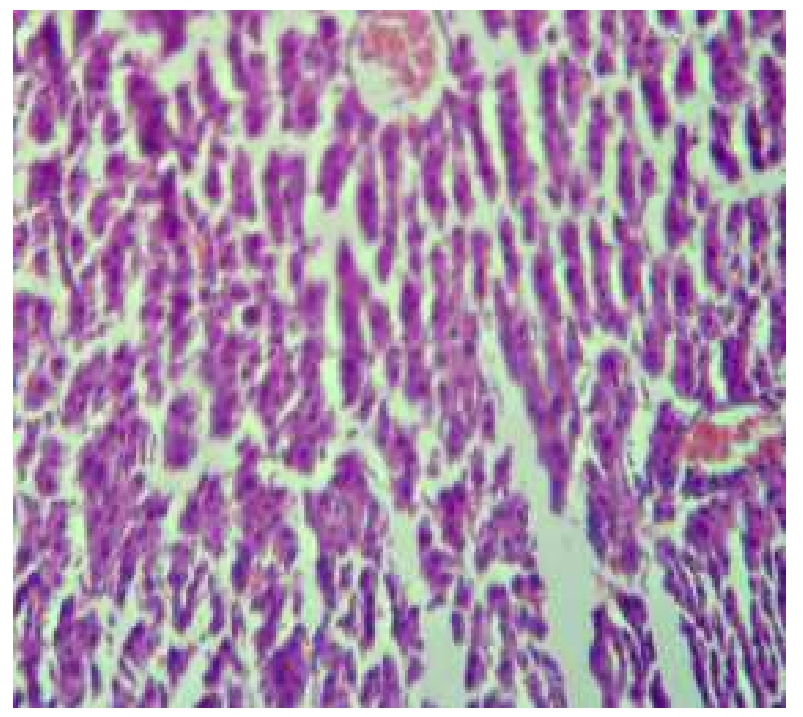

(D) : Lot D $(\mathrm{Cd}-\mathrm{Zn})$; Tissu hépatique apparemment normal. Hépathocytes organisées $(\mathrm{H}-\mathrm{E} \times 40)$.

Figure 2: Examen microscopique du tissu hépatique.

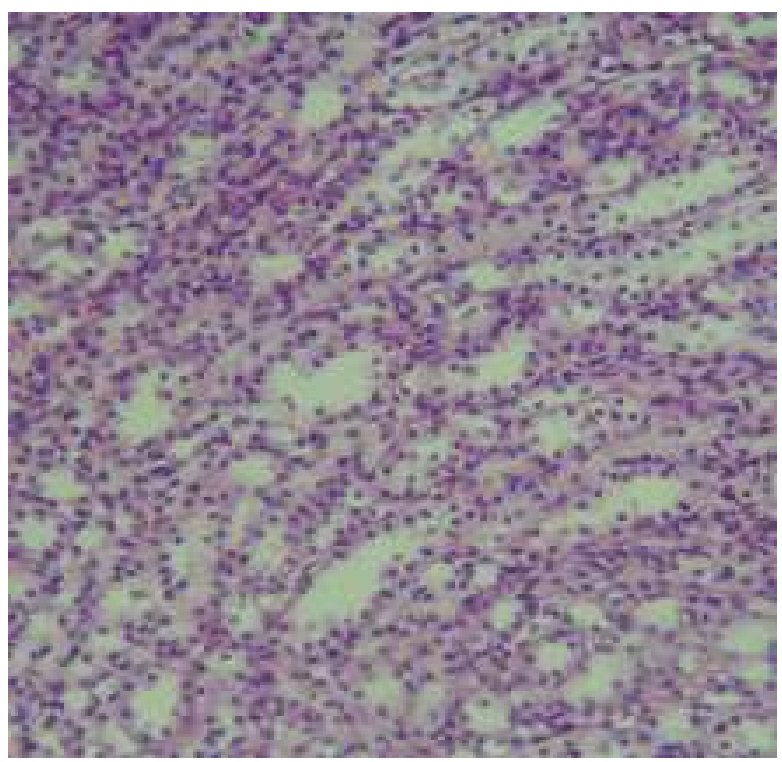

(A) : Lot A (Témoins); Structure normale des tubules et des glomérules dans la zone corticale (Hematoxyline-Eosine $\times$ 40). 
A. BERROUKCHE et al. / Int. J. Biol. Chem. Sci. 8(4): 1796-1807, 2014

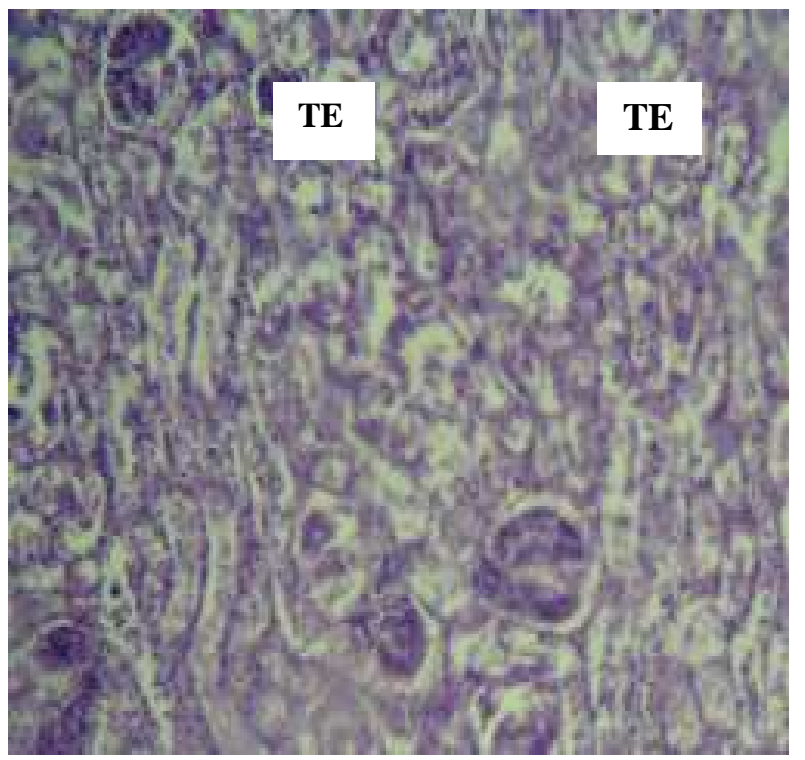

(B) : Lot B $\left(\mathbf{C d S O}_{4}\right)$; Structure tubulaire endommagée [TE] $(\mathrm{H}-\mathrm{E} \times 40)$

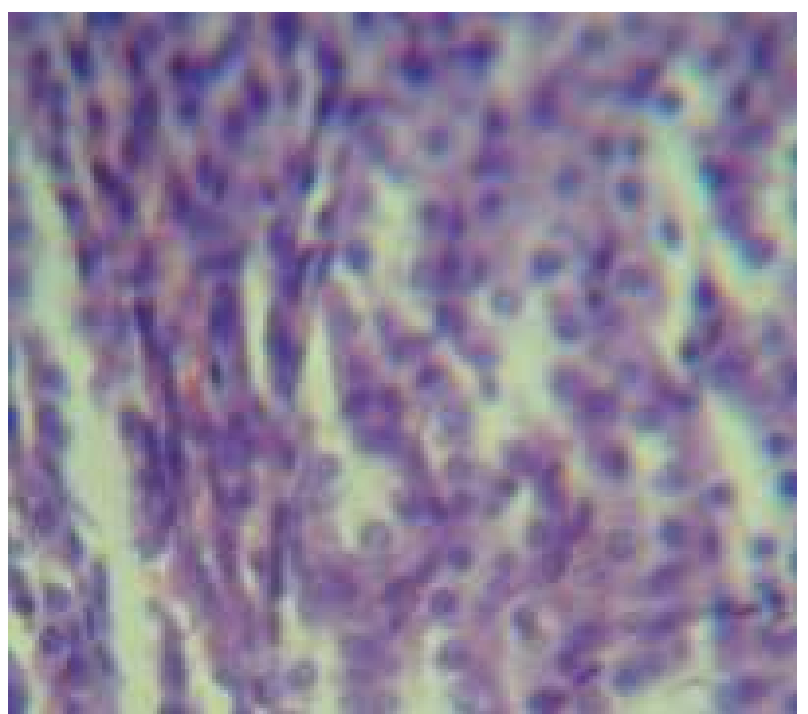

C) : Lot C (ZnCl2) ; Cellules rénales avec des noyaux volumineux [NV] et sombres, nucléoles proéminents ( $\mathrm{HE} \times 40)$. 


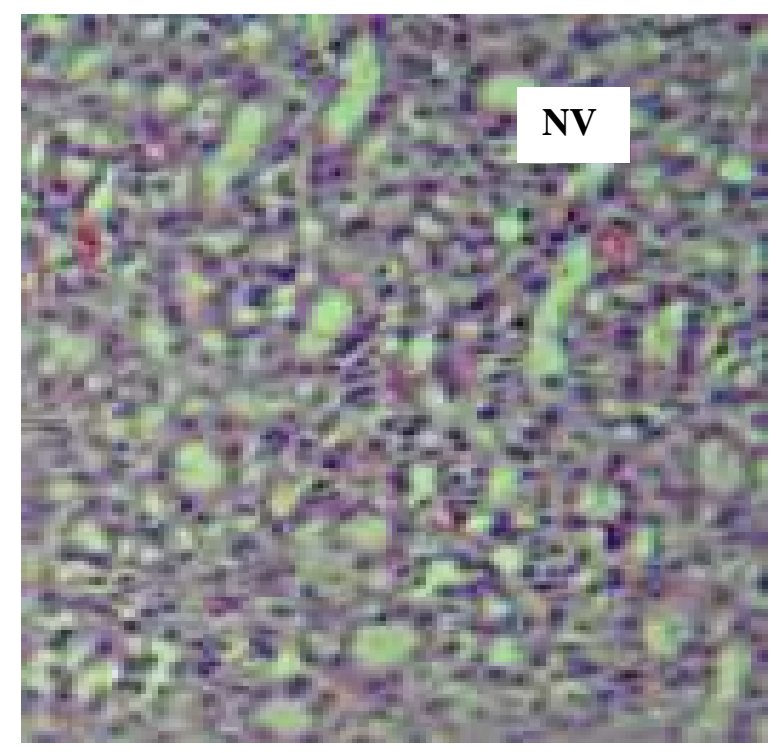

(D) : Lot D (Cd-Zn) ; Parenchyme cellulaire plus ou moins désorganisé, noyaux moins volumineux avec des nucléoles plus sombres $(\mathrm{HE} \times 40)$.

Figure 3 : Examen microscopique du tissu rénal.

Tableau 1 : Poids tissulaires et poids relatifs des organes chez des rats témoins et expérimentés.

\begin{tabular}{lcccc}
\hline & \multicolumn{2}{c}{ Poids des tissus $(\mathbf{g})$} & \multicolumn{2}{c}{ Poids relatifs des tissus (\%) } \\
\cline { 2 - 5 } & Foie & Reins & Foie & Reins \\
\hline Lot A (CdSO4) & $3,93 \pm 1,21$ & $0,73 \pm 0,11$ & $0,03 \pm 0,14$ & $0,00 \pm 0,01$ \\
Lot B (ZnCl2) & $8,66 \pm 4,53$ & $1,16 \pm 0,88$ & $0,07 \pm 0,17$ & $0,00 \pm 0,03$ \\
Lot C (Cd-Zn) & $6,69 \pm 2,78$ & $0,82 \pm 0,45$ & $0,04 \pm 0,18$ & $0,00 \pm 0,02$ \\
Lot D (Témoins) & $10,73 \pm 6,62$ & $1,63 \pm 1,07$ & $0,05 \pm 0,22$ & $0,00 \pm 0,03$ \\
\hline
\end{tabular}

Tableau 2 : Examen macroscopique des tissus prélevés chez les rats témoins et expérimentés.

\begin{tabular}{|c|c|c|c|c|}
\hline \multicolumn{5}{|c|}{ Aspects macroscopiques des tissus prélevés } \\
\hline & Lot $\mathrm{A}\left(\mathrm{CdSO}_{4}\right)$ & Lot $B\left(\mathrm{ZnCl}_{2}\right)$ & Lot C (Cd-Zn) & Lot D (Témoins) \\
\hline \multirow{3}{*}{ Foie } & Zones blanchâtres & $\begin{array}{l}\text { Kystes remplis de } \\
\text { liquide séreux }\end{array}$ & Aspect dur & Aspect \\
\hline & & $\begin{array}{c}\text { Zones blanchâtres et } \\
\text { grisâtres }\end{array}$ & $\begin{array}{l}\text { Changement de } \\
\text { couleur }\end{array}$ & normal \\
\hline & & & $\begin{array}{l}\text { Présence de } \\
\text { kystes }\end{array}$ & \\
\hline Reins normal & Aspect & Normal & Normal & Normal \\
\hline
\end{tabular}




\section{DISCUSSION}

Dans la présente étude, les effets toxiques et cancérigènes $\mathrm{du} \mathrm{Cd}$ sur des rats Wistar, suite à une intoxication chronique par voie orale, ont été évalués. La dose du $\mathrm{Cd}$ $(0,15 \mathrm{mg} / \mathrm{kg}$ poids corporel), utilisée dans cette étude, a eu un effet sur le poids corporel des rats entraînant ainsi sa diminution. Ce résultat concorde avec celui d'une étude américaine qui a utilisé une dose de $\mathrm{Cd}$ un peu plus élevé $(0,25 \mathrm{mg} / \mathrm{kg}$ ) (Newairy et al., 2007). Plusieurs travaux ont signalé l'association $\mathrm{du} \mathrm{Cd}$ à des lésions prolifératives au niveau du tissu rénal. En outre, d'importantes lésions inflammatoires au niveau du foie ont été aussi induites par une dose du Cd.

Les concentrations de $\mathrm{Cd}$ et de $\mathrm{Zn}$ augmentent, chez l'homme, dés la $1^{\text {ere }}$ décennie de sa vie et elles commencent à diminuer modérément à partir de la $7^{\mathrm{e}}$ ou $8^{\mathrm{e}}$ décennie, en fonction de l'âge (Telisman et al., 2000). Cependant, il a été montré que le $\mathrm{Zn}$ peut jouer un rôle protecteur contre les effets toxiques d'une dose élevée de $\mathrm{Cd}$ au niveau des reins en induisant ainsi sa rétention. Le rôle protecteur du $\mathrm{Zn}$ est probablement lié à son effet antioxydant visà-vis $\mathrm{du} \mathrm{Cd}$ et aussi au processus de rétention de ce métal au niveau rénal (Jihen et al., 2010). Les concentrations de Cd sont particulièrement élevées dans la fumée du tabac. C'est pourquoi le taux de $\mathrm{Cd}$ dans les reins des fumeurs est beaucoup plus élevé que chez les non-fumeurs du même âge (Jurczuk et al., 2004). Le Cd pénètre dans notre organisme par les poumons, puis absorbé au niveau des intestins et véhiculé par le sang jusqu'à être déposé principalement au niveau des tissus hépatique et rénal où la métallothionéine (MT), protéine de faible poids moléculaire, joue un rôle majeur dans le métabolisme. La demi-vie biologique de $\mathrm{Cd}$, chez l'homme, peut s'allonger jusqu'à 30 ans et sans aucun doute que son accumulation dans l'organisme a une incidence importante concernant sa toxicité chronique et ses effets cancérogènes. Dans la littérature toxicologique, il est indiqué que le $\mathrm{Cd}$ s'accumule principalement dans le foie et les reins, car ces tissus sont très riches en métallothionéine (MT), il s'agit d'une protéine de liaison du métal présentant ainsi une forte affinité pour le $\mathrm{Cd}$ (Wlostowski et al., 2000).

Les résultats des examens histologiques de cette étude suggèrent que le $\mathrm{Cd}$ pourrait être présent en forte concentration dans ces deux tissus. Les poids élevés des tissus hépatique et rénal pourraient constituer un indice de la présence de ce métal lourd au niveau du cytoplasme de leurs cellules.

Cela peut être expliqué par le fait qu'après absorption $\mathrm{du} \mathrm{Cd}$, ce dernier est accumulé dans le foie où il induit la production du MT pour sa liaison avec cette protéine. Lorsque la concentration hépatique de MT devient insuffisante pour se lier à tous le $\mathrm{Cd}$, ce métal entrâne des lésions membranaires des hépatocytes et favorisant ainsi la libération des complexes Cd-MT dans la circulation sanguine. Arrivé au niveau des reins, les complexes MT-Cd sont filtrés à travers les glomérules puis réabsorbés par les cellules tubulaires proximales (Liu et al., 2000).

Des lésions rénales ont été également observées chez le rat résultant des effets de pression et de l'accumulation de $\mathrm{Cd}$ dans les reins. Une néphropathie induite par le $\mathrm{Cd}$, chez le rat, est caractérisée par des lésions tubulaires et glomérulaire (El Heni et al., 2008). Les zones corticales du rein accumulent sélectivement du $\mathrm{Cd}$ qui est lié à des protéines de faible poids moléculaire donnant naissance au complexe protéique "Cd-Thionéine" (El Heni et al., 2008). 
Dans une étude récente, le complexe Cd-thionéine s'est avérée trés néphrotoxique (El Heni et al., 2008). Les complexes protéine-Cd sont filtrés par les glomérules puis réabsorbés et stockés par les tubules rénaux. Il est suggéré qu'une atteinte du tube rénal est bien la caractéristique d'une néphropathie induite par le $\mathrm{Cd}$ (El Heni et al., 2008). Une étude récente a montré que l'injection de $\mathrm{Cd}$ chez le rat peut provoquer une élévation de l'activité de la rénine plasmatique ainsi qu'une hypertension (Il'yasova et Schwartz, 2005). Chez l'homme, deux études ont indiqué que le $\mathrm{Cd}$ est un facteur causant le cancer du rein (Il'yasova et Schwartz, 2005). Chez les rongeurs, les lésions rénales ou les transformations néoplasiques peuvent être induites par plusieurs autres métaux cancérigènes. Par exemple, le plomb induit des néoplasmes au niveau des cellules rénales tubulaires chez les souris et les rats (Waisberg et al. , 2003). De même, le nickel peut être un initiateur de la transformation cellulaire au niveau de l'épithélium des tubules rénaux chez les rats. Ces lésions peuvent être des facteurs promoteurs déclenchant des tumeurs rénales malignes (Diwan et al., 1989).

\section{Conclusion}

Les résultats de cette étude sont une contribution à la connaissance et l'exploration de la toxicité du $\mathrm{Cd}$ au niveau des tissus hépatique et rénal. La dose modérée du $\mathrm{Cd}$, à $15 \mathrm{mg} / \mathrm{L}$, était suffisante pour générer des lésions prolifératives et des foyers de néoplasie au niveau des différents tissus chez le rat Wistar. Pour le moment, il est recommandé de penser aux risques encourus par l'homme lors de la consommation des aliments riches en $\mathrm{Cd}$ et également dans certains milieux professionnels incriminés pour une forte concentration de $\mathrm{Cd}$. Le $\mathrm{Zn}$ a joué un rôle protecteur vis-à-vis de la toxicité du Cd chez le modèle animal. Cependant, il reste à élucider les mécanismes de cette protection. Des études cas-témoins ou de cohortes sur une population humaine appréciable est souhaitable afin d'évaluer les forces d'association entre le véritable risque d'exposition au $\mathrm{Cd}$ et l'apparition de certains signes pathologiques.

\section{REMERCIEMENTS}

Nous remercions les techniciens du laboratoire de pathologie, les médecins pathologistes et le directeur du laboratoire pour leur contribution à la réalisation des techniques d'examens histologiques en matières de macroscopie et de microscopie ainsi que le rôle cruciale qu'ils ont joué dans la lecture des coupes des échantillons microscopique et l'interprétation des résultats.

\section{REFERENCES}

Brzoska M, Moniuszko-Jakoniuk J. 2000. Interactions between cadmium and zinc in the organism. Food Chem Toxicol., 39: 967-80.

Diwan BA, Ohshima M, Rice JM. 1989. Promotion by sodium barbital of renal cortical and transitional cell tumors, but not intestinal tumors, in F344 rats given methyl(acetoxymethyl)- nitrosamine, and the lack of effect of phenobarbital, amobarbital, or barbituric acid on development of either renal or intestinal tumors. Carcinogenesis, 10: 183-88.

El Heni J, Messaoudi I, Hamouda F, Kerkeni A. 2008. Protective effects of selenium (Se) and zinc $(\mathrm{Zn})$ on cadmium $(\mathrm{Cd})$ toxicity in the liver and kidney of the rat: Histology and $\mathrm{Cd}$ accumulation. Food and Chemical Toxicology, 46: 35223527

Fahmy M, Aly F. 2000. In vivo and in vitro studies on the genotoxicity of cadmium 
chloride in mice. J. Appl. Toxicol., 20: 231-238.

Fernandez E, Gustafson A, Anderson M, Hellman B, Dencker L. 2003. Cadmiuminduced changes in apoptic gene expression blocked by zinc supplementation. Toxicol Sci., 10: 85-99.

Il'yasova D, Schwartz GG. 2005. Cadmium and renal cancer. Toxicol Appl Pharmacol., 207: 179-186.

Jihen EH, Fatima H, Nouha A, Baati T, Imed M, Abdelhamid K. 2010. Cadmium retention increase: a probable key mechanism of the protective effect of zinc on cadmium-induced toxicity in the kidney. Toxicol Lett., 196(2): 104-109.

Jurczuk M, Brzoska MM, MoniuszkoJakoniuk J, Galazyn-Sidorczuk M, Kulikowska-Karpinska E. 2004. Antioxidant enzymes activity and lipid peroxidation in liver and kidney of rats exposed to cadmium and ethanol. Food Chem. Toxicol., 42: 429-438.

Lansdown AB, Sampson B, Rowe E. 2000. Experimental observations in the rat on the influence of cadmium on wound repair. Int. J. Exp. Pathol., 82: 35-41.

Liu J, Qu W, Kadiiska MB. 2009. Role of oxidative stress in cadmium toxicity and carcinogenesis. Toxicol Appl Pharmacol., 238(3): 209-214.

Liu Y, Liu J, Habeebu SM, Waalkes MP, Klaassen CD. 2000. Metallothionein-I/II null mice are sensitive to chronic oral cadmium-induced nephrotoxicity. Toxicol. Sci., 57(1): 167-176.

Newairy AA, El-Sharaky AS, Badreldeen MM, Eweda SM, Sheweita SA. 2007 The hepatoprotective effects of selenium against cadmium toxicity in rats. Toxicology., 242: 23-30
Santon A, Irato P, Medici V, D'Inca R, Albergoni LV, Sturniolo GC. 2003. Effect and possible role of $\mathrm{Zn}$ treatment in LEC rats, an animal model of Wilson's disease. Biochim Biophys Acta, 1637: 91-97.

Silvina MA, Nidia NG, Scardapanec L, Zirulnika F, Martınez D, Gimeneza MS. 2004. Morphological changes and oxidative stress in rat prostate exposed to a non-carcinogenic dose of cadmium. Toxicology Letters, 153: 365-76.

Tandon SK, Singh S, Prasad S, Mathur N. 2001. Hepatic and renal metallothionein induction by an oral equimolar dose of zinc, cadmium or mercury in mice. Food Chem Toxicol, 39: 571-577.

Telisman S, Cvitkovic P, Jurasovic J, Pizent A, Gavella, Rocic B. 2000. Semen quality and reproductive endocrine function in relation to biomarkers of lead, cadmium, zinc, and copperin men. Environ. Health Perspect., 108: 45-53.

UPHS (US Public Health Service). 1985. Guide to the Care and Use of Laboratory Animals. National Institutes of Health: Bethesda.

Waisberg M, Joseph P, Hale B, Beyersmann D.2003. Molecular and cellular mechanisms of cadmium carcinogenesis. Toxicology, 192: 95-117.

Wlostowski T, Krasowska A, LaszkiewiczTiszczenko B. 2000. Dietary cadmium induces histopathological changes despite a sufficient metallothionein level in the liver and kidneys of the bank vole (Clethrionomys glareolus). Comp Biochem PhysiolCToxicol Pharmacol., 126(1): 21-28.

WHO (World Health Organisation). 1992. Environmental Health Criteria, 134 Cadmium. IPCS: Geneva. 